\title{
Differential Lines for Common-Mode Suppression Based in Hybrid Microstrip/CPW Technology
}

\author{
Jesús Martel, Senior Member, IEEE, Armando Fernández-Prieto, Member, IEEE, Aintzane Lujambio, \\ Francisco Medina, Fellow, IEEE, Francisco Mesa, Fellow, IEEE, and Rafael R. Boix, Member, IEEE
}

\begin{abstract}
A symmetrical pair of differential microstrip lines implemented in hybrid microstrip/coplanar waveguide (CPW) technology is proposed. Transmission-line models are used to analyze differential- and common-mode responses, allowing efficient design with minimal optimization effort. The structure behaves as a conventional transmission line pair under differentialmode excitation, whereas asymmetrical coupled transmission line theory has to be applied to characterize commonmode operation. The common mode is strongly suppressed thanks to the introduction of a controllable transmission zero. A two-stage version of the structure is used to increase the common-mode rejection bandwidth. All the electrical parameters of the transmission lines have been obtained using an in-house fast quasi-TEM code. The good agreement between transmissionline models, full-wave simulations and measurements confirms the benefits of the structure and the design procedure.
\end{abstract}

Index Terms-Common-mode suppression, differential lines, hybrid microstrip/CPW structures.

\section{INTRODUCTION}

D IFFERENTIAL signal traces are widely used in highspeed digital circuit applications due to their high immunity to noise and electromagnetic interference. Ideal differential lines should preserve signal integrity for the differential mode (DM) path and provide a high level of common-mode (CM) rejection [1]. A number of printed technology solutions have been reported in the literature involving coupled microstrips with defected ground structure (DGS) [2]-[5], electromagnetic band gap (EBG) [6], nonuniform impedance [7], or multilayered substrates [8]-[10]. In this letter, a pair of differential lines based on the hybrid microstrip/CPW structure shown in Fig.1 is proposed. A CPW resonator (conductor \#3) etched in the ground plane is symmetrically located below a pair of conventional parallelcoupled microstrip lines (conductors \#1 and \#2). It will be

Manuscript received July 26, 2016; accepted October 12, 2016. Date of publication January 3, 2017; date of current version January 6, 2017. This work was supported by the Spanish Ministry of Economy and Competitiveness and European Union FEDER Funds (contract TEC2013-41913-P) and by the Spanish Junta de Andalucía (project P12-TIC-1435).

J. Martel is with the Department of Applied Physics II, ETSA, Av. Reina Mercedes s/n, Seville 41012, Spain.

A. Fernández-Prieto, F. Medina, and R. R. Boix are with the Department of Electronics and Electromagnetism, Faculty of Physics, University of Sevilla, Av. Reina Mercedes s/n, Seville 41012, Spain (e-mail: medina@us.es).

A. Lujambio is with the Skylife Engineering, Parque Tecnológico Aeroespacial de Andalucía, Early Ovington Street, 24, Nave 15-16, Seville 41309, Spain.

F. Mesa is with the Department of Applied Physics I, ETSII, Av. Reina Mercedes s/n, Seville 41012, Spain.

Color versions of one or more of the figures in this paper are available online at http://ieeexplore.ieee.org.

Digital Object Identifier 10.1109/LMWC.2016.2629970

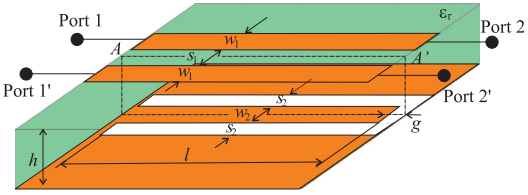

(a)

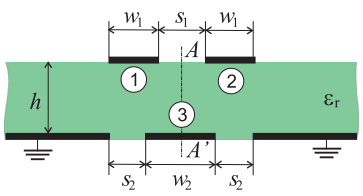

(b)

Fig. 1. (a) 3D layout and (b) cross section of a unit cell of the proposed structure. Two coupled strips are faced to a CPW section (shorted in one end and open-circuited in the other end) etched in the ground plane.

shown by means of a coupled transmission line (TL) analysis that good DM performance and strong CM rejection are provided by this simple configuration. Experimental validation of a two-stage version of the proposed structure is presented to illustrate the design procedure.

\section{COUPled TL MOdels}

A 3D view of the proposed structure is depicted in Fig.1(a) and its cross section is shown in Fig.1(b). The CPW section in the ground plane is short-circuited at one end and opencircuited at the opposite end. From the electrical point of view this structure resembles the one reported in [9]. However, our proposal makes use of a single layer substrate without via holes. The structure in Fig.1(b) supports three quasiTEM modes [11]: an odd mode, with voltage eigenvector $(1,-1,0)$, and two even modes, $E_{\pi}$ and $E_{\mathrm{c}}$, with voltage eigenvectors $\left(1,1,-R_{\pi}\right)$ and $\left(1,1, R_{\mathrm{c}}\right)\left[R_{\pi}, R_{\mathrm{c}}>0\right]$. Under DM operation, the $A A^{\prime}$ symmetry plane (see Fig.1) behaves as an electric wall (EW) in such a way that the $\mathrm{CPW}$ resonator is virtually grounded. Thus, the resulting structure only has a single active conductor (\#1), whose electrical response can be modeled by means of a conventional TL with characteristic parameters $Z_{\text {odd }}$ and $\varepsilon_{\text {odd }}$ (see Fig.2(a)). All-pass behavior for the DM is achieved by enforcing $Z_{\text {odd }}=Z_{0}=50 \Omega$, with $Z_{0}$ being the single-ended reference impedance. The fast quasi-TEM analysis developed in [12] is used to determine the appropriate dimensions of the traces. Under CM operation, the $A A^{\prime}$ plane is a magnetic wall (MW). In such case the resulting structure has two active conductors (\#1 and \#3) and can then be modeled with the coupled asymmetric TL equivalent circuit in Fig.2(b). Following the theory and 


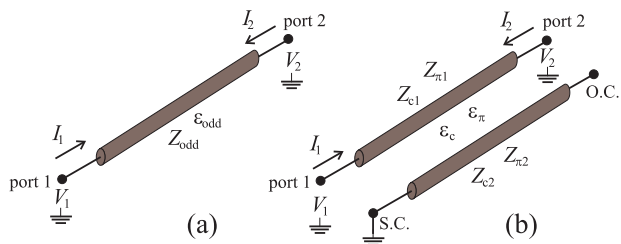

Fig. 2. Equivalent TL circuits for (a) DM operation and (b) CM operation of the structure proposed in Fig.1.

notation in [13], its electrical response can be rigorously described in terms of a quasi-odd $\pi$ mode and a quasieven $c$ mode. The impedances $Z_{\pi 1}$ and $Z_{\mathrm{c} 1}$ correspond to those of the pair of coupled microstrip lines of the three-conductor structure in the modes $E_{\pi}$ (effective dielectric constant $\varepsilon_{\pi}$ ) and $E_{\mathrm{c}}$ (effective dielectric constant $\varepsilon_{\mathrm{c}}$ ). $Z_{\pi 2}$ and $Z_{\mathrm{c} 2}$ are simply twice the impedances corresponding to the $\mathrm{CPW}$ (conductor \#3) of the three-conductor structure in the modes $E_{\pi}$ and $E_{\mathrm{c}}$. Once again, the electrical parameters of the coupled-TL circuit accounting for CM operation are extracted from the analysis of the three-conductor system using the method in [12]. Since the CPW section is terminated with a short-circuit at one end and with an open-circuit at the other end, the CM TL circuit in Fig.2(b) is a two-port circuit. Its impedance matrix can easily be obtained from the elements $z_{\mathrm{ij}}$ of the four-port impedance matrix in [13]

$$
\begin{aligned}
{\left[\begin{array}{l}
V_{1} \\
V_{2}
\end{array}\right] } & =\left[\begin{array}{ll}
Z_{11} & Z_{12} \\
Z_{21} & Z_{22}
\end{array}\right]\left[\begin{array}{l}
I_{1} \\
I_{2}
\end{array}\right] \\
Z_{11} & =z_{11}-\frac{z_{12}^{2}}{z_{22}}, \quad Z_{22}=z_{11}-\frac{z_{13}^{2}}{z_{22}} \\
Z_{12} & =Z_{21}=z_{14}-\frac{z_{12} z_{13}}{z_{22}} \\
z_{11} & =-\mathrm{j} \frac{Z_{\mathrm{c} 1} \operatorname{cotan}\left(\theta_{\mathrm{c}}\right)}{1-R_{\mathrm{c}} / R_{\pi}}-\mathrm{j} \frac{Z_{\pi 1} \operatorname{cotan}\left(\theta_{\pi}\right)}{1-R_{\pi} / R_{\mathrm{c}}} \\
z_{12} & =-\mathrm{j} \frac{Z_{\mathrm{c} 1} R_{\mathrm{c}} \operatorname{cotan}\left(\theta_{\mathrm{c}}\right)}{1-R_{\mathrm{c}} / R_{\pi}}-\mathrm{j} \frac{Z_{\pi 1} R_{\pi} \operatorname{cotan}\left(\theta_{\pi}\right)}{1-R_{\pi} / R_{\mathrm{c}}} \\
z_{13} & =-\mathrm{j} \frac{Z_{\mathrm{c} 1} R_{\mathrm{c}} \operatorname{cosec}\left(\theta_{\mathrm{c}}\right)}{1-R_{\mathrm{c}} / R_{\pi}}-\mathrm{j} \frac{Z_{\pi 1} R_{\pi} \operatorname{cosec}\left(\theta_{\pi}\right)}{1-R_{\pi} / R_{\mathrm{c}}} \\
z_{14} & =-\mathrm{j} \frac{Z_{\mathrm{c} 1} \operatorname{cosec}\left(\theta_{\mathrm{c}}\right)}{1-R_{\mathrm{c}} / R_{\pi}}-\mathrm{j} \frac{Z_{\pi 1} \operatorname{cosec}\left(\theta_{\pi}\right)}{1-R_{\pi} / R_{\mathrm{c}}} \\
z_{22} & =\mathrm{j} \frac{Z_{\mathrm{c} 2} R_{\mathrm{c}} \operatorname{cotan}\left(\theta_{\mathrm{c}}\right)}{R_{\pi}\left(1-R_{\mathrm{c}} / R_{\pi}\right)}+\mathrm{j} \frac{Z_{\pi 2} R_{\pi} \operatorname{cotan}\left(\theta_{\pi}\right)}{R_{\mathrm{c}}\left(1-R_{\pi} / R_{\mathrm{c}}\right)}
\end{aligned}
$$

where $\theta_{\mathrm{c}}=2 \pi f l_{\mathrm{eff}} \sqrt{\varepsilon_{\mathrm{c}}} / c$ and $\theta_{\pi}=2 \pi f l_{\mathrm{eff}} \sqrt{\varepsilon_{\pi}} / c(c$ is the speed of light in vacuum, $f$ is the frequency, and $l_{\text {eff }}=l+\Delta l_{\mathrm{oc}}$; the extra length $\Delta l_{\mathrm{oc}}$ stands for the edge effect associated with the open circuit end of the CPWresonator, which can be approximately calculated using the closed-form expressions in [14]). The coupled-TL circuit in Fig.2(b) presents a transmission zero (TZ) at the frequency $f_{\mathrm{Z}}$ for which $Z_{12}=0$. This frequency can easily be controlled through the choice of the effective length, $l_{\text {eff }}$, of the CPW resonator. $\mathrm{CM}$ rejection bandwidth can also be tuned by adjusting a different geometrical parameter of the structure, as it will be shown later.

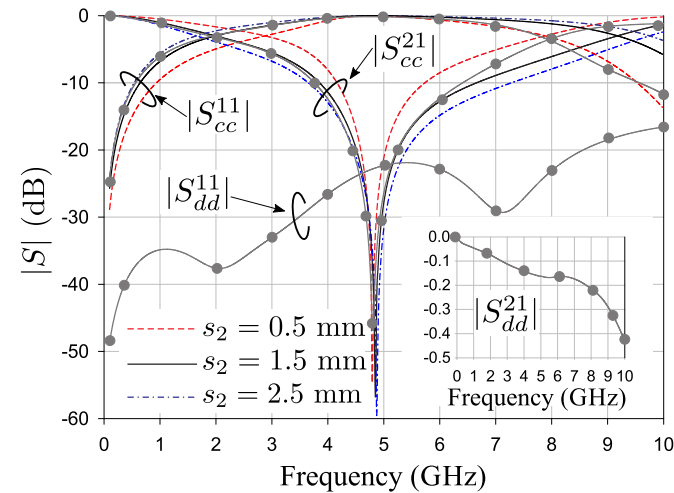

Fig. 3. CM responses (TL model) of the proposed structure for several values of $s_{2}$ (dimensions: $l_{\mathrm{ef}}=12 \mathrm{~mm}, w_{1}=1.1 \mathrm{~mm}, s_{1}=0.4 \mathrm{~mm}$, $\left.w_{2}=2 w_{1}+s_{1}=2.6 \mathrm{~mm}\right)$. FW simulations (DM and $\mathrm{CM}$ ) are included for $s_{2}=1.5 \mathrm{~mm}$

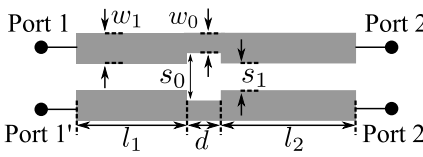

(a)

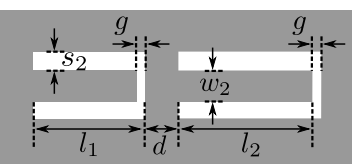

(b)
Fig. 4. (a) Top view and (b) bottom view of the layout of a two-stage structure. Dimensions (in $\mathrm{mm}$ ): $l_{1}=7.4, l_{2}=11.4, g=0.45, w_{1}=1.1$, $s_{1}=0.4, w_{2}=2.6$ and $s_{2}=1.5$.

\section{Design Procedure of a Single Stage}

In order to avoid degradation of the integrity of the differential signal, the design process starts by obtaining the transverse dimensions of the coupled lines [see Fig.1(b)] leading to $Z_{\text {odd }}=50 \Omega$. The parameters of the chosen substrate are $\varepsilon_{\mathrm{r}}=2.43$ and thickness $h=0.483 \mathrm{~mm}$. Since there are four available parameters $\left(w_{1}, s_{1}, w_{2}\right.$ and $\left.s_{2}\right)$ that affect the value of $Z_{\text {odd }}$, additional constraints can be imposed. Since the electric field for DM operation is strongly concentrated in the gap $s_{1}, w_{2}$ has been chosen as $w_{2}=2 w_{1}+s_{1}$ in order to minimize the influence of $s_{2}$ on the DM parameters. With this choice, $Z_{\text {odd }}$ hardly depends on $s_{2}$. Also, in order to avoid problems with fabrication tolerances, a value of $s_{1}=0.4$ $\mathrm{mm}$ has been imposed. Finally, the remaining geometrical parameters are obtained by using the code developed in [12]. The resulting strip widths are $w_{1}=1.10 \mathrm{~mm}$ and $w_{2}=$ $2.60 \mathrm{~mm}$. In Fig. 3 it is plotted the $\mathrm{CM}$ response for the value $l_{\text {eff }}=12 \mathrm{~mm}$ obtained from the coupled-TL model in Fig.2(b) for different values of $s_{2}$. It can be appreciated that $f_{\mathrm{Z}}$ remains practically unchanged around $4.8 \mathrm{GHz}$ when $s_{2}$ is varied ( $f_{\mathrm{Z}}$ mainly depends on $\left.l_{\text {eff }}\right)$. In addition, the higher $s_{2}$, the wider the CM rejection bandwidth. This is because the $\mathrm{CM}$ return currents flowing through the ground plane are more distorted when the value of $s_{2}$ is increased. DM fullwave (FW) simulations are included in Fig. 3 for the case $s_{2}=1.5 \mathrm{~mm}$. The values $g=0.4 \mathrm{~mm}$ and $l=10.65 \mathrm{~mm}$ have been chosen for fine tuning of $f_{\mathrm{Z}}$ (these are the only numerically optimized parameters). Good agreement between the FW and the lossless TL model is observed (DM insertion loss is below $0.5 \mathrm{~dB}$ up to $10 \mathrm{GHz}$ ).

\section{Two-Stage Design and Measurements}

High CM rejection bandwidth can be achieved by using a two-stage structure, as shown in Fig.4. Two CM TZs are 
TABLE I

COMPARISON OF SEVERAL DifFERENTIAL LiNES With CM SUPPRESSION. $\triangle$ IS THE CM STOPBAND FRACTIONAL BANDWIDTH

\begin{tabular}{c|c|c|c|c} 
Ref. & $\begin{array}{c}\text { length } \\
\left(\lambda_{g}\right)\end{array}$ & $\begin{array}{c}\text { width } \\
\left(\lambda_{g}\right)\end{array}$ & $\begin{array}{c}\Delta>20 \mathrm{~dB} \\
(\%)\end{array}$ & $\begin{array}{c}\Delta>30 \mathrm{~dB} \\
(\%)\end{array}$ \\
\hline \hline$[2]$ & 0.85 & 0.43 & 53.3 & 35.6 \\
{$[3]$} & 0.44 & 0.49 & - & - \\
{$[4]$} & 0.64 & 0.13 & 37.7 & 29.6 \\
{$[5]$} & 0.32 & 0.22 & 67 & 33.3 \\
{$[6]$} & 1.58 & 0.32 & 70 & 63 \\
{$[10]$} & 0.43 & 0.19 & 15 & - \\
This work & 0.58 & 0.14 & 61 & 48 \\
\hline
\end{tabular}

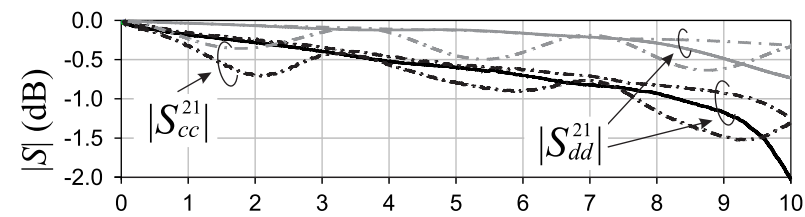

(a)

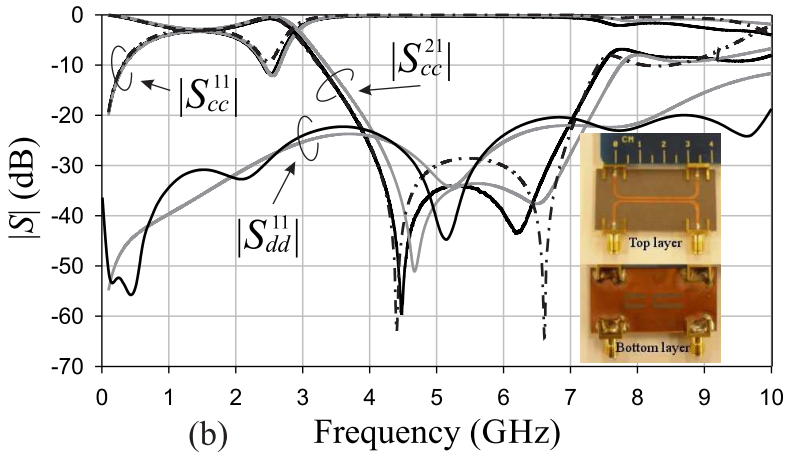

Fig. 5. (a) DM and CM response of the conventional differential lines (dot and dashed lines) and the DM-insertion loss of the new proposed $\mathrm{CM}$ rejection filter (solid line). Gray lines stand for $\mathrm{FW}$ simulations and black lines stand for measured data. (b) DM-return loss and CM response of the new structure. Dot and dashed lines stand for the TL circuit model, gray lines stand for FW simulations and black lines stand for measured data.

now possible. The two stages are connected through a pair of coupled microstrips of length $d=2.90 \mathrm{~mm}$, which have to be included in the cascade TL circuit. The new coupled section behaves like a TL of impedance $Z_{\text {odd }}^{d}(\mathrm{DM})$ or $Z_{\text {even }}^{d}(\mathrm{CM})$. The transverse dimensions of this coupled pair must be chosen in such a way that $Z_{\text {odd }}^{d}=Z_{\text {odd }}$ to ensure DM all-pass response. We have used $w_{0}=w_{1}$ and $s_{0}=s_{1}$ and the matching condition has been reasonably fulfilled. The circuit depicted in Fig. 4 has been fabricated and measured with a four-port Agilent ANA (PNA-E8363B with N4420B). Simulated and measured DM and CM responses are shown in Fig.5. Results for the conventional coupled-lines (with solid ground plane) are included for comparison purposes. Note please the improvement in CM rejection for the new design when compared with the conventional one, and the similar DM performance in both designs except for a small degradation at high frequencies due to radiation loss through the slotted ground plane. The CM TL model response is included for comparison (the effective lengths of each stage are $l_{\mathrm{eff} 1}=8 \mathrm{~mm}$ and $l_{\mathrm{eff} 2}=12 \mathrm{~mm}$ ). A very good agreement between simulations and measurements is observed considering the reduced optimization effort carried out. Differences between simulated and measured results are probably caused by fabrication tolerances and associated misalignment between top and bottom planes, which is specially relevant in balanced circuits due to mode conversion. A comparison between our proposal and other contributions found in the literature is given in Table I. According to this table, structure size and CM stopband fractional bandwidth are found to be competitive in spite of the extreme simplicity of the new design.

\section{CONCLUSION}

A hybrid microstrip/CPW differential pair is presented and accurately modeled using asymmetric coupled TL theory, thus avoiding numerical optimization. The proposed structure exhibits competitive DM and CM performance concerning bandwidth, CM rejection level and size when compared with other more complex implementations. Measurements, fullwave and circuit simulations agree reasonably well.

\section{REFERENCES}

[1] T.-W. Weng, C.-H. Tai, C.-H. Chen, D.-H. Han, and T.-L. Wu, "Synthesis model and design of a common-mode bandstop filter (CM-BSF) with all pass characteristics for high speed differential signals," IEEE Trans. Microw. Theory Techn., vol. 62, no. 8, pp. 1647-1656, Aug. 2014.

[2] W.-T. Liu, C.-H. Tsai, T.-W. Han, and T.-L. Wu, "An embedded common-mode suppression filter for $\mathrm{GHz}$ differential signals using periodic defected ground plane," IEEE Microw. Wireless Compon. Lett., vol. 18, no. 4, pp. 248-250, Apr. 2008.

[3] S.-J. Wu, C.-H. Tsai, T.-L. Wu, and T. Itoh, "A novel wideband commonmode suppression filter for gigahertz differential signals using coupled patterned ground structure," IEEE Trans. Microw. Theory Techn., vol. 57, no. 4, pp. 848-855, Apr. 2009.

[4] J. Naqui et al., "Common mode suppression in microstrip differential lines by means of complementary split ring resonators: Theory and applications," IEEE Trans. Microw. Theory Techn., vol. 60, no. 10, pp. 3023-3033, Oct. 2012.

[5] A. Fernández-Prieto et al., "Dual-band differential filter using broadband common-mode rejection artificial transmission line," Prog. Electromag. Res., vol. 139, pp. 779-797, Apr. 2013.

[6] J. H. Choi, P. W. C. Hon, and T. Itoh, "Dispersion analysis and design of planar electromagnetic bandgap ground plane for broadband commonmode suppression," IEEE Microw. Wireless Compon. Lett., vol. 24, no. 11, pp. 772-775, Apr. 2014.

[7] P. Vélez, J. Bonache, and F. Martín, "Differential microstrip lines with common-mode suppression based on electromagnetic band gaps," IEEE Antennas Wireless Propag. Lett., vol. 18, pp. 40-43, Jan. 2015.

[8] C.-H. Tsai and T.-L. Wu, "A broadband and miniaturized common-mode filter for gigahertz differential signals based on negative-permittivity metamaterials," IEEE Trans. Microw. Theory Techn., vol. 58, no. 1, pp. 195-202, Jan. 2010.

[9] G.-H. Shiue, C.-M. Hsu, C.-L. Lou, and C.-F. Su, "A comprehensive investigation of a common-mode filter for gigahertz differential signals using quarter-wave resonators," IEEE Trans. Compon. Packag. Manuf. Technol., vol. 4, no. 1, pp. 134-144, Jan. 2014.

[10] A. Fernández-Prieto et al., "Common-mode suppression for balanced bandpass filters in multilayer liquid crystal polymer technology," IET Microw. Antennas Propag., vol. 9, no. 12, pp. 1249-1253, Sep. 2015.

[11] V. K. Tripathi, "On the analysis of symmetrical three-line microstrip circuits," IEEE Trans. Microw. Theory Techn., vol. 25, no. 9, pp. 726-729, Sep. 1977.

[12] J. Martel and F. Medina, "A suitable integral equation for the quasiTEM analysis of hybrid strip/slot-like structures," IEEE Trans. Microw. Theory Techn., vol. 49, no. 1, pp. 224-227, Jan. 2001.

[13] V. K. Tripathi, "Asymmetric coupled transmission lines in an inhomogeneous medium," IEEE Trans. Microw. Theory Techn., vol. 23, no. 9, pp. 734-739, Sep. 1975.

[14] M. Mao, R. Wu, C.-H. Chen, and C.-H. Lin, "Characterization of coplanar waveguide open end capacitance-theory and experiment," IEEE Trans. Microw. Theory Techn., vol. 42, no. 6, pp. 1016-1024, Jun. 1994. 